\title{
Younger Age Is Associated with Poorer Survival in Patients with Signet-Ring Cell Carcinoma of the Colon without Distant Metastasis
}

\author{
Ben Huang, Mengdong Ni, Chen Chen, Yang Feng, and Sanjun Cai \\ Department of Colorectal Surgery, Fudan University Shanghai Cancer Center, 270 Dongan Road, Shanghai 20032, China
}

Correspondence should be addressed to Sanjun Cai; csjfuscc@163.com

Received 18 July 2016; Revised 2 October 2016; Accepted 19 October 2016

Academic Editor: Daniele Marrelli

Copyright (C) 2016 Ben Huang et al. This is an open access article distributed under the Creative Commons Attribution License, which permits unrestricted use, distribution, and reproduction in any medium, provided the original work is properly cited.

\begin{abstract}
Background. In general, younger age is associated with better survival in patients with colon cancer. In this study, we aim to analyze the impact of age on cancer-specific survival (CSS) in patients with signet-ring cell carcinoma (SRCC) of the colon, a particularly aggressive type of colon cancer. Methods. Information on patients with SRCC of the colon with no distant metastasis was extracted from the US Surveillance, Epidemiology, and End Results (SEER) database. An X-tile plot was used to determine the optimal cutoff age at diagnosis. Results. A total of 776 patients were included in data analysis. The X-tile program revealed an optimal cutoff at 35 years of age. A higher percentage of stage III disease and a higher percentage of N2 disease were observed in patients $\leq 35$ years of age. The multivariate Cox proportional model demonstrated that patients $\leq 35$ years of age were more likely to have a poorer survival outcome compared with patients aged $>35$ years (HR 1.411, 95\% CI 1.032-1.929, and $P=0.031$ ). Conclusion. In contrast to the association of younger age with better survival in colon cancer patients, younger age ( $\leq 35$ years) is associated with poorer survival outcome in patients with SRCC of the colon without distant metastasis.
\end{abstract}

\section{Introduction}

Signet-ring cell carcinoma (SRCC) is a rare but distinct subtype of colon cancer and makes up roughly $1 \%$ of all colorectal cancer cases [1-5]. It is a very aggressive form of colon cancer $[1,6]$ and is associated with poor biological behaviors, including poor differentiation $[1,2]$, perineural or lymphovascular invasion [1,7], and lymph node involvement $[4,8]$. From a molecular biological perspective, colorectal SRCC is also recognized as a unique tumor entity, inclining to have high levels of BRAF mutations, KRAS mutations, and CpG island methylation $[7,9,10]$.

The incidence rate of colorectal cancer has been decreasing over the past three decades [11], mostly due to the decline in adenocarcinoma [12]. By contrast, the incidence rate of SRCC has slightly increased over the past few years, probably due to increasing recognition of the disease in daily practice [12]. Hitherto, the prognostic determinants of SRCC remain largely undefined. Lee et al. [13] studied 19 patients with primary SRCC of the colon and rectum who underwent curative surgery and found that these patients had a poorer prognosis compared to other types of colorectal cancer. Belli et al. [14] reported a retrospective series of 22 colorectal SRCC patients and they found that these patients were diagnosed at an advanced stage and that, among others, tumor site and TNM stage had significant effect on survival.

Colorectal SRCC is noted for a significantly higher proportion of younger patients compared to non-SRCC colorectal cancer. A large population-based study, including 196,757 cases of colorectal cancer, showed that $7.7 \%$ of SRCC patients were under the age of 45 years, whereas only $2.7 \%$ of colorectal adenocarcinoma patients were younger than 45 years of age [3]. Li et al. [15] evaluated 69,835 patients with colorectal cancer in the Surveillance, Epidemiology, and End Results (SEER) database and found a significantly higher rate of SRCC in patients younger than 40 years of age $(2.8 \%)$ than those older than 40 years of age $(0.8 \%)$.

For patients with colorectal cancer in general, younger age is associated with better or comparable survival [15-19]. Currently, scant knowledge is available on the prognostic value of younger age in SRCC of the colon. Considering the aggressive biological behavior and extremely poor oncologic 
outcomes of SRCC of the colon, we hypothesized that younger patients with this particular histological subtype of colon cancer may harbor a biologically aggressive phenotype and have worse prognosis than older patients. The SEER database contains 18 cancer registries covering $26 \%$ of the US population, collecting and providing cancer incidence and survival data. To address this hypothesis, we analyzed the cancer-specific survival (CSS) of patients with SRCC of the colon without distant metastasis in the SEER database and determined the prognostic value of age and other variables for CSS. Subgroup analysis was conducted in stage I/II and stage III SRCC of the colon.

\section{Materials and Methods}

2.1. Patient Selection. We extracted the demographic and clinicopathological records of invasive colon cancer patients from January 1988 to December 2011 from the SEER database (http://seer.cancer.gov/, April 2013 release). Patients meeting the following criteria were included in the current analysis: (1) age between 18 and 74 years at the time of diagnosis; (2) pathologically confirmed SRCC of the colon; (3) known depth of invasion and lymph node status; (4) at least 12 lymph nodes harvested; (5) colon cancer surgically resected with pathology specimen; (6) known survival time and cause of death. Patients were excluded if (1) they underwent only local tumor excision; (2) diagnosis of colon cancer was obtained from death certificate or by autopsy; (3) there was distant metastasis of colon cancer (AJCC stage M0); (4) there were other concurrent malignancies. The study protocol was reviewed and approved by the Fudan University Shanghai Cancer Center Ethical Committee and Institutional Review Board. Informed consent is not applicable since the study was based on a publicly available database (the SEER database).

2.2. Outcome Measures. Data on the following variables were retrieved from the SEER database: gender, race, age at diagnosis, years of diagnosis, pathological grade, number of primary lesions, number of lymph nodes harvested and positive lymph nodes (N0, N1, and N2), and depth of local invasion (T1, T2, T3, and T4), American Joint Committee on Cancer (AJCC) TNM stage, radiation sequence with surgery, follow-up duration, and SEER cancer-specific death classification. All cases were restaged by the 7th AJCC TNM staging system. In this study, the right colon refers to the cecum, the ascending colon, the hepatic flexure of the colon, and the transverse colon, whereas the left colon refers the splenic flexure of the colon, the descending colon, and the sigmoid colon.

2.3. Statistical Analysis. CSS was the primary endpoint of our study and was calculated from the time of diagnosis to the time of colon cancer-specific death. Patients who died from other causes or were alive at the last follow-up were censored. We employed the X-tile software (http://medicine .yale.edu/lab/rimm/research/software.aspx) (Yale School of Medicine, CT, USA) to determine the optimal cutoff age at diagnosis using the minimum $P$ values from log-rank chisquared statistics for stratification of patients into the high or low risk group [20]. The X-tile plots allow a single, global assessment of every possible way of dividing a population into low and high risk for survival. In the X-tile analysis, data are displayed in the $x$-axis where each point reflects a different cutoff point. The intensity of color in the grid indicates the strength of association, in this study, between age at diagnosis and CSS. Patient data stratified by the cutoff age were summarized using cross-tabulation, and the distributions were compared using chi-squared tests.

Survival curves were plotted using the Kaplan-Meier method. In addition, log-rank test was used for univariate analysis and variables with a $P$ value $<0.1$ were entered into the Cox proportion hazard regression model. Multivariate Cox regression analyses were used to generate adjusted hazards ratios (HR) and their corresponding 95\% confidence intervals (CIs). Subgroup analyses were conducted in stage I/II and stage III SRCC of the colon. All statistical analyses were performed using SPSS version 20.0 for Windows (SPSS Inc., Chicago, IL, USA). A two-sided $P$ value of less than 0.05 was accepted as statistically significant.

\section{Results}

3.1. Demographic and Baseline Characteristics of SRCC Patients. Seven hundred seventy-six cases (432 men and 344 women) from the SEER database were included in data analysis. The sample was predominantly Caucasian (82.1\%). Patient demographic and clinicopathological features are shown in Table 1 . Their mean age at diagnosis was 56 years and $90.0 \%$ (698) of them were aged more than 35 years. The median follow-up duration was 27 (interquartile range, 12 to 64$)$ months. The primary lesion was in the right colon in $78.9 \%$ of the cases. Most patients (89.7\%) had poor or undifferentiated SRCC. Furthermore, 400 (51.6\%) subjects had metastasis in more than three lymph nodes $\left(\mathrm{N}_{2}\right)$. TNM stage III cases were the most common, accounting for $74.9 \%$ (581), and stage I cases the least common 4.5\% (35). Moreover, compared to patients $>35$ years of age, patients $\leq 35$ years of age had a significantly higher proportion of non-Caucasians (35.9\% versus $15.9 \%, P<0.001)$ and a markedly greater percentage of $\mathrm{N}_{2}$ SRCC $(73.1 \%$ versus $49.1 \%, P<0.001)$ and stage III SRCC $(89.7 \%$ versus $73.2 \%, P=0.006)$ (Table 1$)$.

3.2. Impact of Age on Survival of SRCC Patients. In this study, 318 (41.0\%) colon cancer-specific deaths were observed. The one-year CSS stood at $83.8 \%$, the three-year CSS at $59.8 \%$, and the five-year CSS at $52.2 \%$ for the entire cohort (Figure 1(a)). Kaplan-Meier analyses showed that younger patients had poorer CSS than older patients $(P<0.001$ at a cutoff of 30 years of age, Figure 1(b); $P<0.001$ at a cutoff of 35 years of age, Figure 1(c); $P=0.015$ at a cutoff of 40 years of age, Figure $1(\mathrm{~d}))$. An X-tile analysis indicated optimal cutoff age at 35 years (Figure 2). When 35 years of age was used as the optimal cutoff to stratify patient survival, the Kaplan-Meier analysis revealed that patients $\leq 35$ years of age had poorer CSS compared with patients $>35$ years and the 5-year CSS was $31.1 \%$ and $54.9 \%$ in patients $\leq 35$ years and patients > 35 years $(P<0.001)$ (Figure $1(\mathrm{c}))$. An analysis using the multivariate Cox proportional model further demonstrated 
TABLE 1: Demographic and baseline characteristics of patients with nonmetastatic signet-ring cell carcinoma of the colon from the SEER database and stratification by age at diagnosis.

\begin{tabular}{|c|c|c|c|c|}
\hline Characteristics & All patients & $\leq 35$ years & $>35$ years & $\begin{array}{c}P \text { value } \\
\left(\text { Pearson } \chi^{2}\right)\end{array}$ \\
\hline$N(\%)$ & $776(100.0)$ & $78(10.0)$ & $698(90.0)$ & \\
\hline Median follow-up, months & 27 & 23 & 28 & \\
\hline Male gender, $n(\%)$ & $432(55.7)$ & $42(53.8)$ & $390(55.9)$ & $0.732(0.12)$ \\
\hline Years of diagnosis, $n(\%)$ & & & & $0.029(4.76)$ \\
\hline 1988-2003 & $309(39.8)$ & $40(51.3)$ & $269(38.5)$ & \\
\hline 2004-2011 & $467(60.2)$ & $38(48.7)$ & $429(61.5)$ & \\
\hline Primary site, $n(\%)$ & & & & $0.001(10.31)$ \\
\hline Right colon & $612(78.9)$ & $50(64.1)$ & $562(80.5)$ & \\
\hline Left colon & $164(21.1)$ & $28(35.9)$ & $136(19.5)$ & \\
\hline Race, $n(\%)$ & & & & $<0.001(19.84)$ \\
\hline White & $637(82.1)$ & $50(64.1)$ & $587(84.1)$ & \\
\hline Black & $83(10.7)$ & $16(20.5)$ & $67(9.6)$ & \\
\hline Others $^{\mathrm{a}}$ & $56(7.1)$ & $12(15.4)$ & $44(6.3)$ & \\
\hline Pathology grade, $n(\%)$ & & & & $0.987(0.001)$ \\
\hline Well/moderate & $80(10.3)$ & $8(10.3)$ & $72(10.3)$ & \\
\hline Poor/undifferentiated & $696(89.7)$ & $70(89.7)$ & $626(89.7)$ & \\
\hline Tumor size, $n(\%)$ & & & & $0.123(2.49)$ \\
\hline$\leq 4.0 \mathrm{~cm}$ & $249(32.1)$ & $19(24.4)$ & $230(33.0)$ & \\
\hline$>4.0 \mathrm{~cm}$ & $527(67.9)$ & $59(75.6)$ & $468(67.0)$ & \\
\hline LNH, $n(\%)$ & & & & $0.023(5.19)$ \\
\hline$\leq 18$ & $343(44.2)$ & $25(32.1)$ & $318(45.6)$ & \\
\hline$>18$ & $433(55.8)$ & $53(67.9)$ & $380(54.4)$ & \\
\hline T stage, $n(\%)$ & & & & $0.164(5.11)$ \\
\hline $\mathrm{T} 1$ & $24(3.1)$ & $2(2.5)$ & $22(3.1)$ & \\
\hline $\mathrm{T} 2$ & $31(4.0)$ & $1(1.3)$ & $30(4.3)$ & \\
\hline T3 & $453(58.4)$ & $40(51.3)$ & $413(59.2)$ & \\
\hline $\mathrm{T} 4$ & $268(34.5)$ & $35(44.9)$ & $233(33.4)$ & \\
\hline $\mathrm{N}$ stage, $n(\%)$ & & & & $<0.001(17.08)$ \\
\hline N0 & $195(25.1)$ & $8(10.2)$ & $187(26.8)$ & \\
\hline N1 & $181(23.3)$ & $13(16.7)$ & $168(24.1)$ & \\
\hline $\mathrm{N} 2$ & $400(51.6)$ & $57(73.1)$ & $343(49.1)$ & \\
\hline TNM stage, $n(\%)$ & & & & $0.006(10.27)$ \\
\hline I & $35(4.5)$ & $1(1.3)$ & $34(4.9)$ & \\
\hline II & $160(20.6)$ & $7(9.0)$ & $153(21.9)$ & \\
\hline III & $581(74.9)$ & $70(89.7)$ & $511(73.2)$ & \\
\hline
\end{tabular}

LNH: number of lymph nodes harvested.

${ }^{a}$ Native Americans, Asians, Pacific Islanders, and unknown.

that age was an independent prognostic factor for CSS. Patients $\leq 35$ years of age were more likely to have a poorer survival outcome compared with patients aged $>35$ years (HR 1.411, 95\% CI 1.032-1.929, and $P=0.031$ ) (Table 3).

Our univariate analysis showed that, apart from age, primary site $(P=0.049), \mathrm{N}$ and $\mathrm{T}$ stage $(P<0.001$ in both) were prognostic determinants of CSS (Table 2). Analysis using a multivariate Cox proportional model suggested that, in addition to age at diagnosis, $\mathrm{N}$ and $\mathrm{T}$ stages were independent determinants of CSS of SRCC patients from the
SEER database $(P<0.001$ in both) (Table 3). Compared to N0 stage patients, N2 stage patients were more than 6 times more likely to succumb to SRCC (HR 6.392, 95\% CI 4.102-9.961, and $P<0.001$ ). However, multivariate analysis revealed no significant difference between SRCC patients with T1 stage and T4 stage (HR: 3.988; 95\% CI: 0.970-16.391, $P=0.055)$.

Kaplan-Meier curves for stage I, stage II, and stage III SRCC patients were shown in Figure 3(a). In the planned subgroup analysis, the association between young age (35 


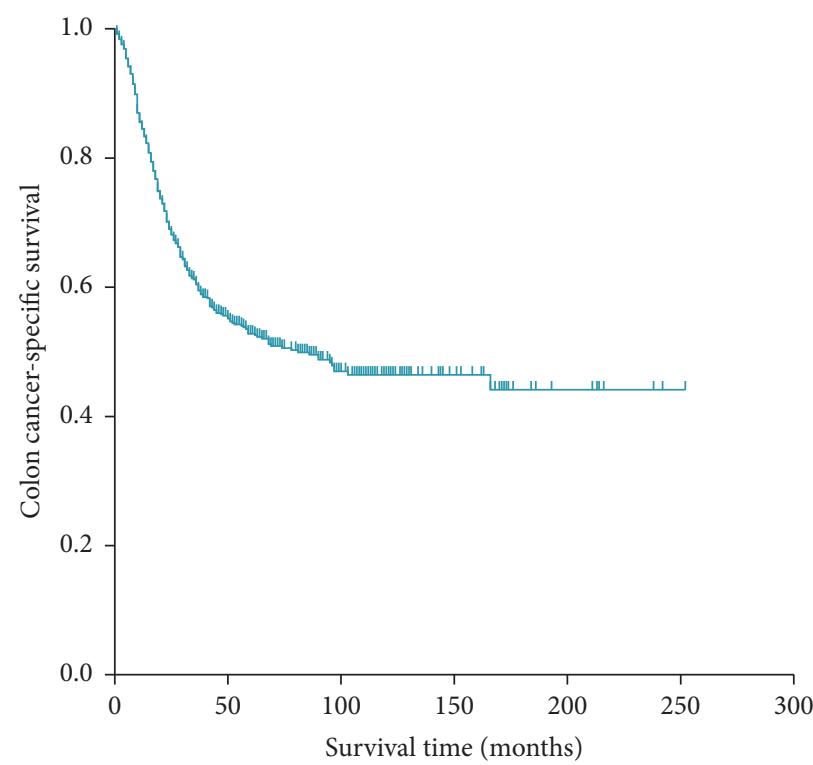

(a)

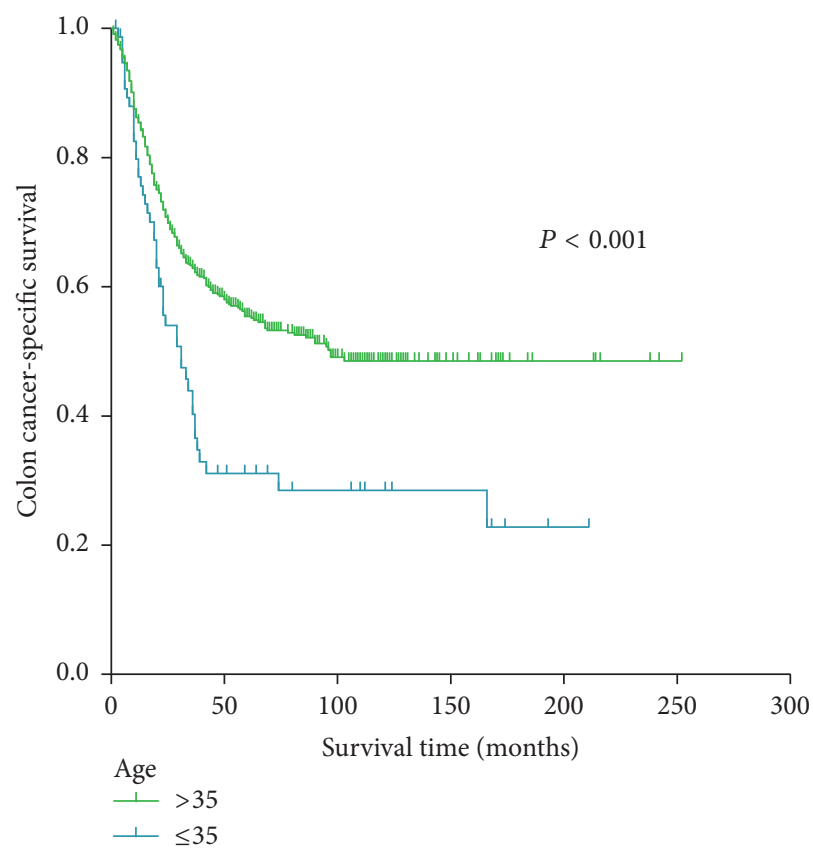

(c)

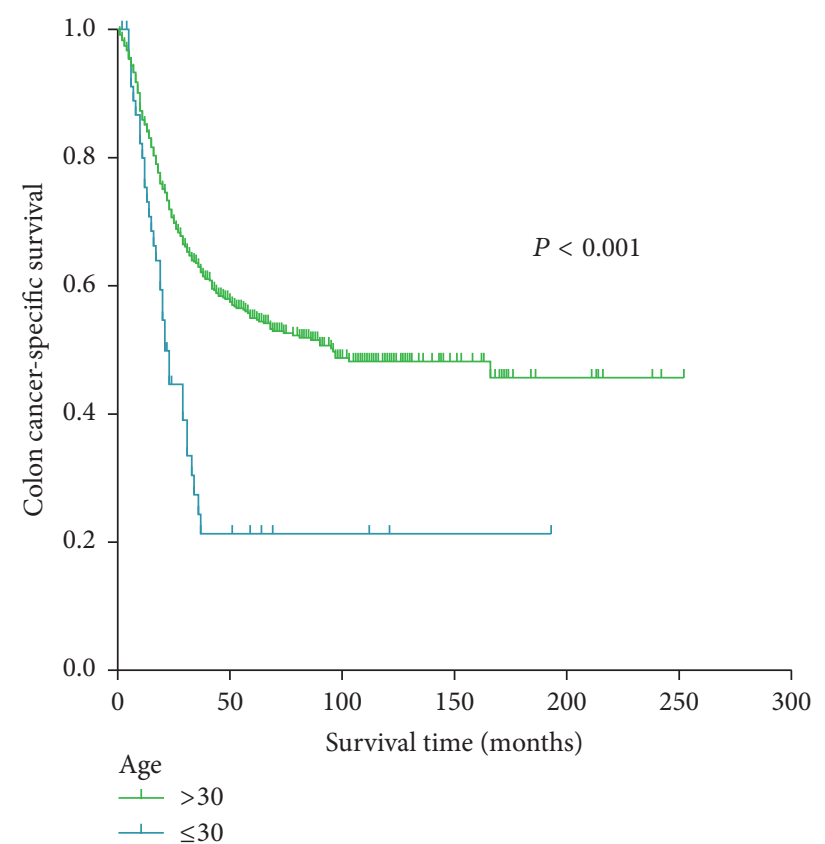

(b)

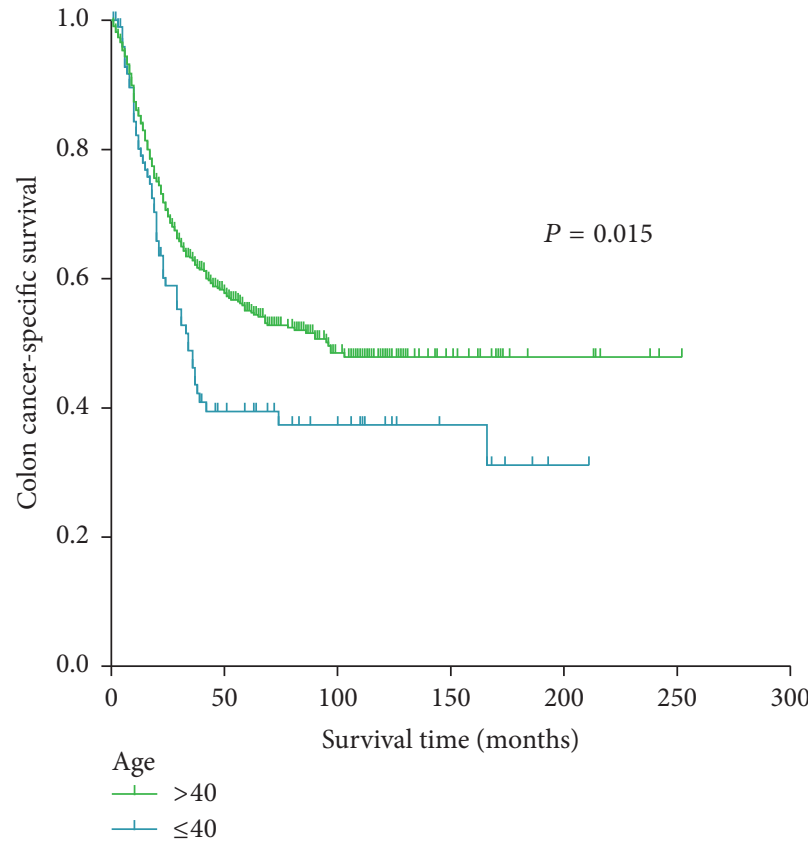

(d)

FIGURE 1: (a) The Kaplan-Meier survival curve for patients with nonmetastatic signet-ring cell carcinoma (SRCC) of the colon from the Surveillance, Epidemiology, and End Results (SEER) cohort. Kaplan-Meier curves for patients with nonmetastatic SRCC of the colon stratified by (b) 30 years of age, (c) 35 years of age, and (d) 40 years of age.

years as the cutoff) and poor CSS was not observed in patients with stage I/II SRCC $(P=0.788)$ (Figure 3(b)). By contrast, stage III SRCC patients $\leq 35$ years of age had a significantly lower CSS compared with those $>35$ years $(P=0.008)$, the 5 -year CSS was $23.4 \%$ and $42.5 \%$ in patients $\leq 35$ years and patients $>35$ years (Figure 3(c)). Multivariate Cox proportional model was further conducted in stage III SRCC patients. Consistently, age $(P=0.034)$, $\mathrm{N}$ stage $(P<0.001)$, and $\mathrm{T}$ stage $(P=0.003)$ were independent prognostic determinants of CSS for stage III SRCC patients (Table 4). Compared with stage III SRCC $>35$ years of age, patients $\leq 35$ years of age were more likely to have a poorer CSS (HR, 1.416, 95\% CI 1.027-1.951, $P=0.034$ ) (Table 4). 


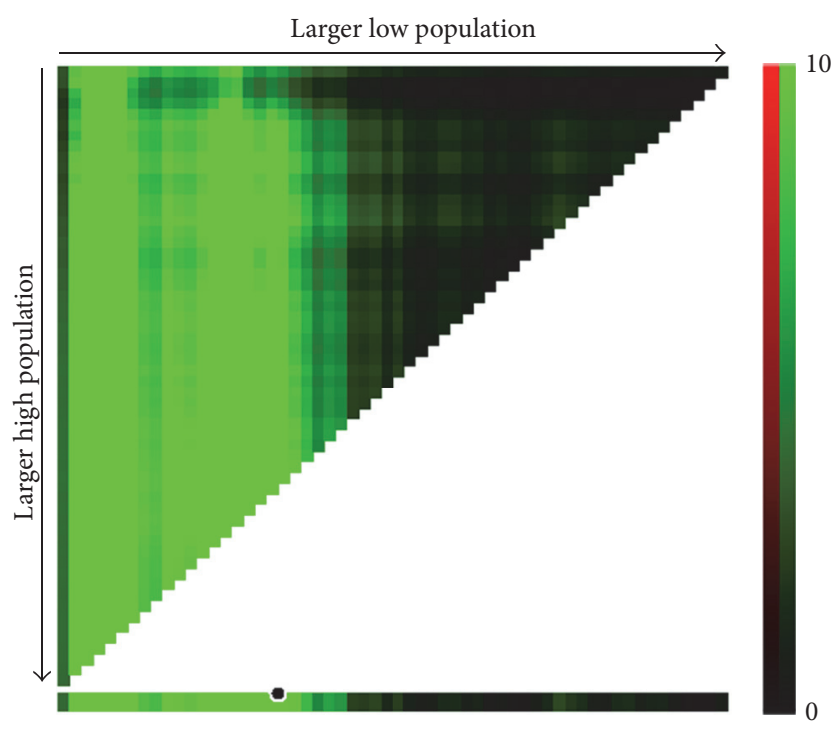

FIgURE 2: X-tile plot of age at diagnosis in the SEER cohort of patients with SRCC of the colon. $x$-axis represents all age cutoff values from low to high (left to right) to define the high and low subset. Brighter pixels indicate stronger association between age at diagnosis and cancer-specific survival (CSS). The plot shows the brightest pixel (marked by the black circle) when the study cohort is divided into the high and low subset using 35 years of age as the cutoff. Green color indicates continuous direct association between increasing age at diagnosis and greater CSS.

\section{Discussion}

Colorectal SRCC often presents as advanced tumors [4, 21], with metastases at multiple sites, especially peritoneal carcinomatosis $[8,21-23]$. The disease is typically diagnosed at an advanced TNM stage [2-4] and has a dismal clinical outcome. However, the prognostic determinants of colorectal SRCC remain largely undefined. In the current study, we analyzed the demographic and clinicopathological variables of 776 cases of SRCC of the colon without distant metastasis in the SEER database. We demonstrated a five-year CSS of $52.2 \%$ for the SRCC cohort from the SEER database. The major finding is poor survival in young patients, with an optimal cutoff at 35 years of age. The most recent studies, however, reported that age showed no correlation with survival in patients with colorectal SRCC. Wang et al. [24] analyzed 59 patients with colorectal SRCC and found that age (40 years old as cutoff value) was not a prognostic factor for survival. Kakar and Smyrk [25] reported that age (70 years old as cutoff value) was not significantly associated with survival in a study including 72 patients with SRCC of the colorectum.

Colorectal SRCC tends to occur in younger patients compared to non-SRCC colorectal cancer. It remains controversial whether younger colorectal cancer patients had comparable or better prognosis versus older patients $[15,18$, $19,26-28]$. We found that younger patients with SRCC of the colon without distant metastasis ( $\leq 35$ years of age) fared poorer in CSS compared with older patients ( $>35$ years of age). A preplanned subgroup analysis confirmed the association of young age with poor outcome in patients with stage
TABLE 2: Univariate analyses of determinants of cancer-specific survival (CSS) of patients with nonmetastatic signet-ring cell carcinoma of the colon.

\begin{tabular}{|c|c|c|c|}
\hline Variable & 5-year CSS & Log rank $\chi^{2}$ & $P$ value \\
\hline Sex & & 0.466 & 0.495 \\
\hline Male & $51.5 \%$ & & \\
\hline Female & $53.4 \%$ & & \\
\hline Years of diagnosis & & 1.801 & 0.180 \\
\hline $1988-2003$ & $50.5 \%$ & & \\
\hline 2004-2011 & $53.7 \%$ & & \\
\hline Race & & 3.169 & 0.205 \\
\hline White & $53.7 \%$ & & \\
\hline Black & $43.4 \%$ & & \\
\hline Other $^{\mathrm{a}}$ & $50.2 \%$ & & \\
\hline Pathology grade & & 1.503 & 0.138 \\
\hline Well/moderate & $58.4 \%$ & & \\
\hline Poor/undifferentiated & $52.5 \%$ & & \\
\hline $\mathrm{LNH}$ & & 0.168 & 0.682 \\
\hline$\leq 18$ & $51.2 \%$ & & \\
\hline$>18$ & $53.5 \%$ & & \\
\hline Tumor size $(\mathrm{cm})$ & & 1.816 & 0.178 \\
\hline$\leq 4.0$ & $54.8 \%$ & & \\
\hline$>4.0$ & $51.9 \%$ & & \\
\hline Primary site & & 3.859 & 0.049 \\
\hline Right colon & $54.9 \%$ & & \\
\hline Left colon & $43.3 \%$ & & \\
\hline Age at diagnosis (year) & & 13.121 & $<0.001$ \\
\hline$\leq 35$ & $31.1 \%$ & & \\
\hline$>35$ & $54.9 \%$ & & \\
\hline $\mathrm{N}$ stage & & 148.599 & $<0.001$ \\
\hline N0 & $88.3 \%$ & & \\
\hline N1 & $61.2 \%$ & & \\
\hline N2 & $30.0 \%$ & & \\
\hline T stage & & 63.528 & $<0.001$ \\
\hline $\mathrm{T} 1$ & $90.4 \%$ & & \\
\hline $\mathrm{T} 2$ & $84.1 \%$ & & \\
\hline $\mathrm{T} 3$ & $59.8 \%$ & & \\
\hline $\mathrm{T} 4$ & $30.2 \%$ & & \\
\hline
\end{tabular}

LNH: number of lymph nodes harvested.

${ }^{a}$ Includes Native American, Asian, Pacific Islander, and unknown.

III SRCC but not stage I/II disease. Also, a markedly higher percentage of N2 SRCC and a markedly higher percentage of stage III SRCC were observed in younger patients ( $\leq 35$ years of age). Younger patients may mistakenly believe that they are unlikely to harbor malignant tumors and consequently seek medical attention at a more advanced stage of SRCC. Equally likely, physicians may miss malignancy in younger SRCC patients due to lowered suspicion, thus missing the best time of treatment. These phenomena may partially contribute to our findings. Also, the late occurrence of clinical symptoms in patients with colorectal SRCC may lead to delay in diagnosis $[29,30]$. 
TABLE 3: Multivariate analyses of determinants of cancer-specific survival (CSS) of patients with nonmetastatic signet-ring cell carcinoma of the colon.

\begin{tabular}{lccc}
\hline \multirow{2}{*}{ Variables } & \multicolumn{3}{c}{ Multivariate analysis } \\
& HR & $95 \%$ CI & $P$ value \\
\hline $\begin{array}{l}\text { Primary site } \\
\quad \text { Right colon }\end{array}$ & 1 & Reference & 0.696 \\
$\quad$ Left colon & 0.950 & $0.732-1.231$ & \\
Age at diagnosis (year) & & & 0.031 \\
$\quad$ 35 & 1 & Reference & \\
s35 & 1.411 & $1.032-1.929$ & \\
N stage & & & $<0.001$ \\
N0 & 1 & Reference & \\
N1 & 2.977 & $1.840-4.817$ & $<0.001$ \\
N2 & 6.392 & $4.102-9.961$ & $<0.001$ \\
T stage & & & $<0.001$ \\
T1 & 1 & Reference & \\
T2 & 1.406 & $0.257-7.707$ & 0.695 \\
T3 & 2.458 & $0.602-10.042$ & 0.210 \\
T4 & 3.988 & $0.970-16.391$ & 0.055 \\
\hline
\end{tabular}

HR: hazard ratio; CI: confidence interval.

The unfathomed distinct genetic basis of young-onset SRCC of the colon may contribute to the poor survival outcomes. As a subtype of colorectal cancer with poor survival, SRCC has intrinsic genetic events that are responsible for its highly malignant nature [1]. Colorectal SRCC has more frequent BRAF mutations and MLH1 loss, less frequent 18q LOH, and lower COX2 levels [31], when compared with non-SRCC colorectal cancer. MSI-H status is closely associated with signet-ring cell differentiation with rates ranging from 43 to $86 \%$ in different studies [32-34]; however, no recognized rate has been reported yet due to small sample size. Likewise, young-onset colorectal cancer also has distinct genetic basis. Many studies have reported a higher frequency of MSI positive colorectal cancers in very young patients [35-37]. Morris et al. [35] showed that, besides MSI, colorectal cancer in young patients correlated well with various molecular features of tumors, such as higher rates of TP-53 mutation and lower rates of BRAF and KRAS mutations. However, the exact genetic features of youngonset SRCC of the colon have been rarely reported. A small number of reports of genetic changes in young colorectal SRCC patients and young patients with SRCC in other body sites such as the stomach are available [38-41]; therefore, further comparative studies of larger patient sample sizes are needed to delineate the genetic peculiarity of young-onset SRCC of the colon.

Based on the data from the SEER database, our study explores the prognostic role of age in determining survival outcomes of patients with SRCC of the colon. Since SRCC is a rare histological cancer subtype, current knowledge was mostly derived from single institution studies based on small population sizes $[6,23]$. We utilized the SEER database to ensure a large sample size, and, to be specific, our study included a total of 776 patients. However, there are still several
TABLE 4: Multivariate survival analyses of determinants of cancerspecific survival (CSS) of patients with stage III signet-ring cell carcinoma of the colon.

\begin{tabular}{lccc}
\hline \multirow{2}{*}{ Variables } & \multicolumn{3}{c}{ Multivariate analysis } \\
& HR & $95 \%$ CI & $P$ value \\
\hline $\begin{array}{l}\text { Primary site } \\
\quad \text { Right colon }\end{array}$ & 1 & Reference & 0.651 \\
$\quad$ Left colon & 0.940 & $0.721-1.227$ & \\
Age at diagnosis (year) & & & 0.034 \\
$\quad$ 35 & 1 & Reference & \\
$\leq 35$ & 1.416 & $1.027-1.951$ & \\
N stage & & & $<0.001$ \\
N1 & 1 & Reference & \\
N2 & 2.170 & $1.628-2.894$ & \\
T stage & & & 0.003 \\
T1 & 1 & Reference & \\
T2 & 1.438 & $0.149-13.830$ & 0.753 \\
T3 & 2.472 & $0.345-17.723$ & 0.368 \\
T4 & 3.721 & $0.518-26.753$ & 0.192 \\
\hline HR: hazards ratio: CI: confidence interval. & &
\end{tabular}

HR: hazards ratio; CI: confidence interval.

limitations in our study. Although SEER is a large populationbased database, the stratification by tumor stage reduced the number of subjects in each study group and statistical power. One remarkable limitation is that the homogeneity of our study population may be impaired by the long study period because there have been rapid developments in colon cancer screening, diagnosing, imaging, and treating during the years from 1988 to 2011. An additional limitation is that some important patient- and disease-related information cannot be obtained from the SEER database, such as intestinal obstruction or penetration, comorbidities, surgical margin status, and data on adjuvant chemotherapy. Furthermore, the SEER database does not have records on family history or molecular biology information; however, such information may be a valuable addition to current data and may help clarify and further understand the risk factors for youngonset SRCC of the colon.

\section{Conclusion}

In conclusion, our analysis indicated that, in contrast to the association of younger age with better survival in colon cancer patients, younger age is associated with a worse survival in patients with SRCC of the colon without distant metastasis, particularly in patients with stage III disease. Further studies are warranted to uncover potential molecular and genetic characteristics of young patients with SRCC of the colon.

\section{Competing Interests}

The authors declare that there are no competing interests regarding the publication of this paper. 


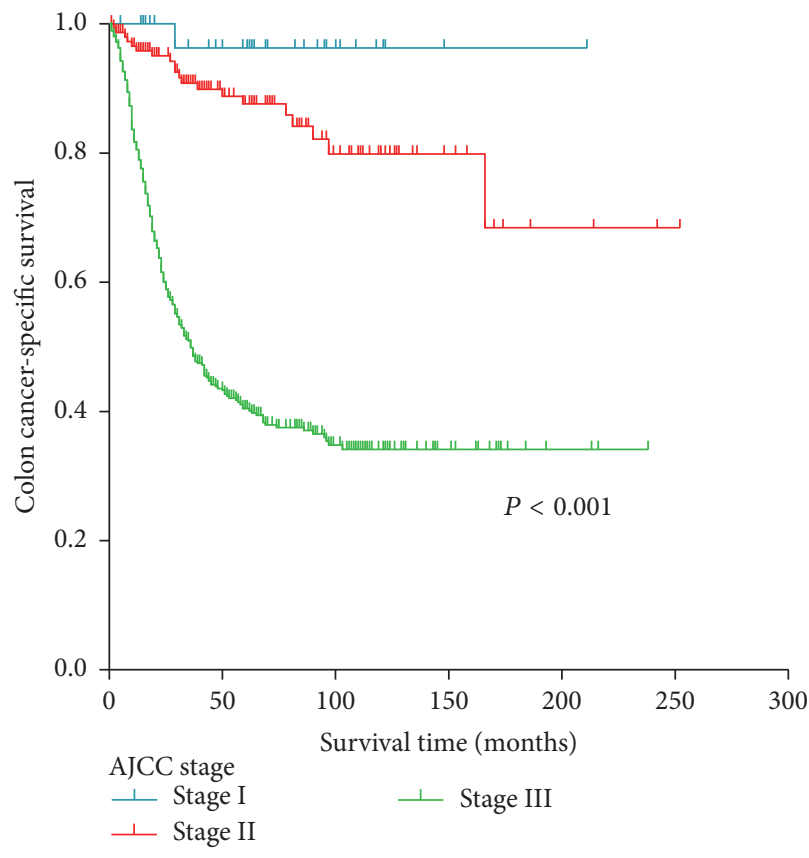

(a)

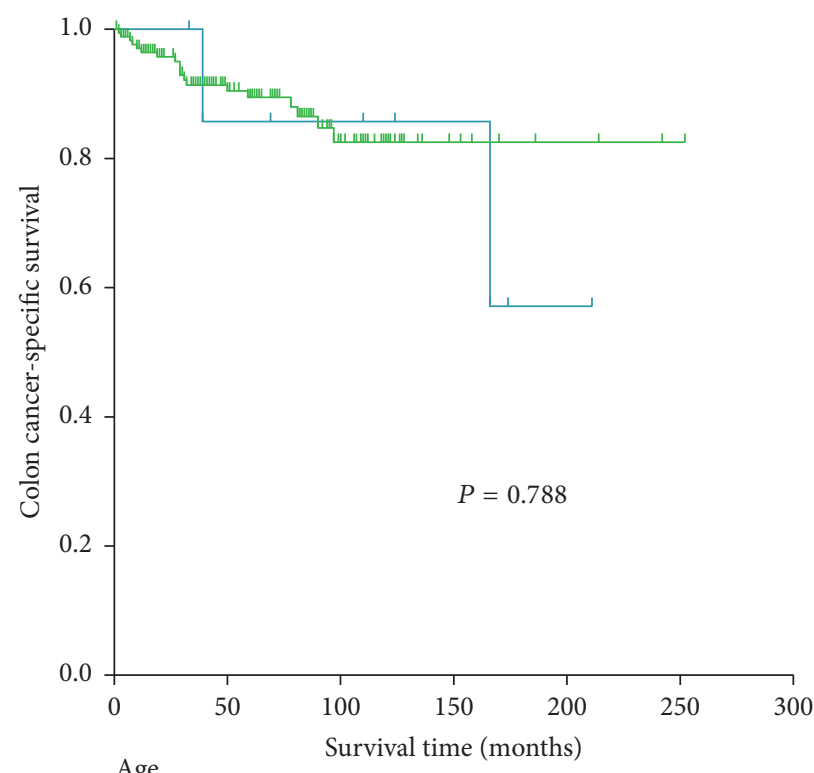

Age

35

(b)

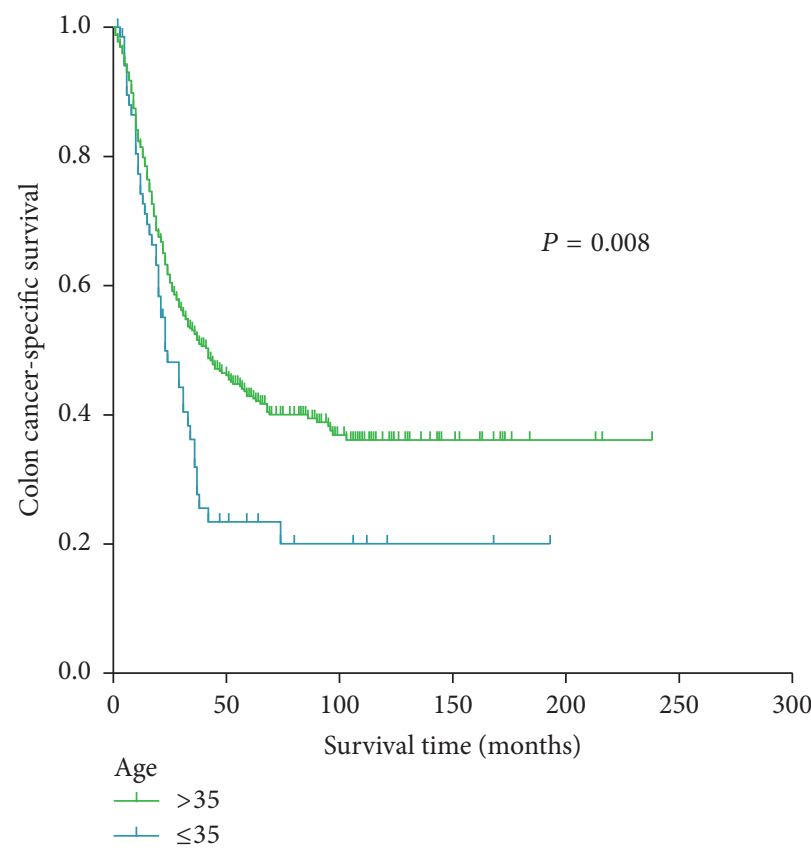

(c)

FIgURE 3: (a) Kaplan-Meier curves for patients with nonmetastatic SRCC of the colon stratified by TNM stage. Kaplan-Meier curves for patients with (b) stage I/II and (c) stage III SRCC of the colon stratified by age at diagnosis ( $\leq 35$ or $>35$ years).

\section{Authors' Contributions}

Ben Huang and Mengdong Ni contributed equally to this work.

\section{Acknowledgments}

The authors would like to thank SEER program for public access to the database.

\section{References}

[1] U. Nitsche, A. Zimmermann, C. Späth et al., "Mucinous and signet-ring cell colorectal cancers differ from classical adenocarcinomas in tumor biology and prognosis," Annals of Surgery, vol. 258, no. 5, pp. 775-783, 2013.

[2] J. R. Hyngstrom, C.-Y. Hu, Y. Xing et al., "Clinicopathology and outcomes for mucinous and signet ring colorectal adenocarcinoma: analysis from the National Cancer Data Base," Annals of Surgical Oncology, vol. 19, no. 9, pp. 2814-2821, 2012. 
[3] N. Hugen, R. H. Verhoeven, V. E. Lemmens et al., "Colorectal signet-ring cell carcinoma: benefit from adjuvant chemotherapy but a poor prognostic factor," International Journal of Cancer, vol. 136, no. 2, pp. 333-339, 2015.

[4] M.-H. Chew, S.-A. E. Yeo, Z.-P. Ng et al., "Critical analysis of mucin and signet ring cell as prognostic factors in an Asian population of 2,764 sporadic colorectal cancers," International Journal of Colorectal Disease, vol. 25, no. 10, pp. 1221-1229, 2010.

[5] W. Song, S.-J. Wu, Y.-L. He et al., "Clinicopathologic features and survival of patients with colorectal mucinous, signetring cell or non-mucinous adenocarcinoma: experience at an institution in southern China," Chinese Medical Journal, vol. 122, no. 13, pp. 1486-1491, 2009.

[6] S. Arifi, O. Elmesbahi, and A. A. Riffi, "Primary signet ring cell carcinoma of the colon and rectum," Bulletin du Cancer, vol. 102, no. 10, pp. 880-888, 2015.

[7] D. J. Hartman, M. N. Nikiforova, D. T. Chang et al., "Signet ring cell colorectal carcinoma: a distinct subset of mucin-poor microsatellite-stable signet ring cell carcinoma associated with dismal prognosis," The American Journal of Surgical Pathology, vol. 37, no. 7, pp. 969-977, 2013.

[8] T. Makino, T. Tsujinaka, H. Mishima et al., "Primary signet-ring cell carcinoma of the colon and rectum: report of eight cases and review of 154 Japanese cases," Hepato-Gastroenterology, vol. 53, no. 72, pp. 845-849, 2006.

[9] V. Gopalan, R. A. Smith, Y.-H. Ho, and A. K.-Y. Lam, "Signetring cell carcinoma of colorectum-current perspectives and molecular biology," International Journal of Colorectal Disease, vol. 26, no. 2, pp. 127-133, 2011.

[10] S. Kakar, G. Deng, T. C. Smyrk, L. Cun, V. Sahai, and Y. S. Kim, "Loss of heterozygosity, aberrant methylation, BRAF mutation and KRAS mutation in colorectal signet ring cell carcinoma," Modern Pathology, vol. 25, no. 7, pp. 1040-1047, 2012.

[11] R. L. Siegel, K. D. Miller, and A. Jemal, "Cancer statistics, 2016," CA: Cancer Journal for Clinicians, vol. 66, no. 1, pp. 7-30, 2016.

[12] H. Kang, J. B. O'Connell, M. A. Maggard, J. Sack, and C. Y. Ko, "A 10 -year outcomes evaluation of mucinous and signet-ring cell carcinoma of the colon and rectum," Diseases of the Colon and Rectum, vol. 48, no. 6, pp. 1161-1168, 2005.

[13] H.-S. Lee, J. S. Soh, S. Lee et al., "Clinical features and prognosis of resectable primary colorectal signet-ring cell carcinoma," Intestinal Research, vol. 13, no. 4, pp. 332-338, 2015.

[14] S. Belli, H. O. Aytac, E. Karagulle, H. Yabanoglu, F. Kayaselcuk, and S. Yildirim, "Outcomes of surgical treatment of primary signet ring cell carcinoma of the colon and rectum: 22 cases reviewed with literature," International Surgery, vol. 99, no. 6, pp. 691-698, 2014.

[15] Q. Li, G. Cai, D. Li, Y. Wang, C. Zhuo, and S. Cai, "Better longterm survival in young patients with non-metastatic colorectal cancer after surgery, an analysis of 69,835 patients in SEER database," PLoS ONE, vol. 9, no. 4, Article ID e93756, 2014.

[16] R. Wang, M. J. Wang, and J. Ping, "Clinicopathological features and survival outcomes of colorectal cancer in young versus elderly: a population-based cohort study of SEER 9 registries data (1988-2011)," Medicine (Baltimore), vol. 94, no. 35, Article ID e1402, 2015.

[17] A. Murata, T. Akiyoshi, M. Ueno et al., "Clinicopathological characteristics of young patients with sporadic colorectal cancer," Surgery Today, vol. 46, no. 10, pp. 1166-1175, 2016.

[18] M. Li, J.-Y. Li, A.-L. Zhao, and J. Gu, "Do young patients with colorectal cancer have a poorer prognosis than old patients?" Journal of Surgical Research, vol. 167, no. 2, pp. 231-236, 2011.
[19] V. S. Schellerer, S. Merkel, S. C. Schumann et al., "Despite aggressive histopathology survival is not impaired in young patients with colorectal cancer: CRC in patients under 50 years of age," International Journal of Colorectal Disease, vol. 27, no. 1, pp. 71-79, 2012.

[20] R. L. Camp, M. Dolled-Filhart, and D. L. Rimm, "X-tile: a new bio-informatics tool for biomarker assessment and outcomebased cut-point optimization," Clinical Cancer Research, vol. 10, no. 21, pp. 7252-7259, 2004.

[21] J.-S. Chen, P.-S. Hsieh, J.-M. Chiang et al., "Clinical outcome of signet ring cell carcinoma and mucinous adenocarcinoma of the colon," Chang Gung Medical Journal, vol. 33, no. 1, pp. 51-57, 2010.

[22] N. Hugen, C. J. H. van de Velde, J. H. W. de Wilt, and I. D. Nagtegaal, "Metastatic pattern in colorectal cancer is strongly influenced by histological subtype," Annals of Oncology, vol. 25, no. 3, Article ID mdt591, pp. 651-657, 2014.

[23] S.-Y. Tung, C.-S. Wu, and P.-C. Chen, "Primary signet ring cell carcinoma of colorectum: an age- and sex-matched controlled study," The American Journal of Gastroenterology, vol. 91, no. 10, pp. 2195-2199, 1996.

[24] R. Wang, X. Ma, Y. Li et al., “The characteristics and prognostic effect of E-cadherin expression in colorectal signet ring cell carcinoma," PLoS ONE, vol. 11, no. 8, Article ID e0160527, 2016.

[25] S. Kakar and T. C. Smyrk, "Signet ring cell carcinoma of the colorectum: correlations between microsatellite instability, clinicopathologic features and survival," Modern Pathology, vol. 18, no. 2, pp. 244-249, 2005.

[26] M. C. Taylor, D. Pounder, N. H. Ali-Ridha, A. Bodurtha, and E. C. MacMullin, "Prognostic factors in colorectal carcinoma of young adults," Canadian Journal of Surgery, vol. 31, no. 3, pp. 150-153, 1988.

[27] J. C. Cusack, G. G. Giacco, K. Cleary et al., "Survival factors in 186 patients younger than 40 years old with colorectal adenocarcinoma," Journal of the American College of Surgeons, vol. 183, no. 2, pp. 105-112, 1996.

[28] K. Marble, S. Banerjee, and L. Greenwald, "Colorectal carcinoma in young patients," Journal of Surgical Oncology, vol. 51, no. 3, pp. 179-182, 1992.

[29] B. S. Ooi, Y. H. Ho, K. W. Eu, and F. S. Choen, "Primary colorectal signet-ring cell carcinoma in Singapore," ANZ Journal of Surgery, vol. 71, no. 12, pp. 703-706, 2001.

[30] K.-I. Fu, Y. Sano, S. Kato et al., "Primary signet-ring cell carcinoma of the colon at early stage: a case report and a review of the literature," World Journal of Gastroenterology, vol. 12, no. 21, pp. 3446-3449, 2006.

[31] S. Ogino, M. Brahmandam, M. Cantor et al., "Distinct molecular features of colorectal carcinoma with signet ring cell component and colorectal carcinoma with mucinous component," Modern Pathology, vol. 19, no. 1, pp. 59-68, 2006.

[32] J. Alexander, T. Watanabe, T.-T. Wu, A. Rashid, S. Li, and S. R. Hamilton, "Histopathological identification of colon cancer with microsatellite instability," American Journal of Pathology, vol. 158, no. 2, pp. 527-535, 2001.

[33] J. K. Greenson, J. D. Bonner, O. Ben-Yzhak et al., "Phenotype of microsatellite unstable colorectal carcinomas: welldifferentiated and focally mucinous tumors and the absence of dirty necrosis correlate with microsatellite instability," The American Journal of Surgical Pathology, vol. 27, no. 5, pp. 563$570,2003$.

[34] J. Shia, N. A. Ellis, P. B. Paty et al., "Value of histopathology in predicting microsatellite instability in hereditary nonpolyposis 
colorectal cancer and sporadic colorectal cancer," The American Journal of Surgical Pathology, vol. 27, no. 11, pp. 1407-1417, 2003.

[35] M. Morris, C. Platell, and B. Iacopetta, "A population-based study of age-related variation in clinicopathological features, molecular. Markers and outcome from colorectal cancer," Anticancer Research, vol. 27, no. 4, pp. 2833-2838, 2007.

[36] J. T. Liang, K. C. Huang, A. L. Cheng, Y. M. Jeng, M. S. Wu, and S. M. Wang, "Clinicopathological and molecular biological features of colorectal cancer in patients less than 40 years of age," British Journal of Surgery, vol. 90, no. 2, pp. 205-214, 2003.

[37] S. M. Farrington, A. J. McKinley, A. D. Carothers et al., "Evidence for an age-related influence of microsatellite instability on colorectal cancer survival," International Journal of Cancer, vol. 98 , no. 6, pp. 844-850, 2002.

[38] A. R. Brooks-Wilson, P. Kaurah, G. Suriano et al., "Germline E-cadherin mutations in hereditary diffuse gastric cancer: assessment of 42 new families and review of genetic screening criteria," Journal of Medical Genetics, vol. 41, no. 7, pp. 508-517, 2004.

[39] P. Guilford, J. Hopkins, J. Harraway et al., "E-cadherin germline mutations in familial gastric cancer," Nature, vol. 392, no. 6674, pp. 402-405, 1998.

[40] S. A. Gayther, K. L. Gorringe, S. J. Ramus et al., "Identification of germ-line E-cadherin mutations in gastric cancer families of European origin," Cancer Research, vol. 58, no. 18, pp. 40864089, 1998.

[41] R. S. Van der Post, I. P. Vogelaar, F. Carneiro et al., "Hereditary diffuse gastric cancer: updated clinical guidelines with an emphasis on germline CDH1 mutation carriers," Journal of Medical Genetics, vol. 52, no. 6, pp. 361-374, 2015. 


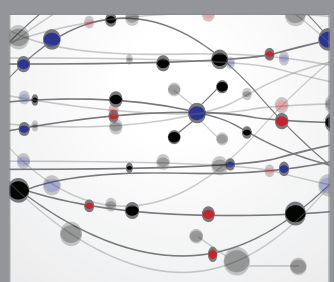

The Scientific World Journal
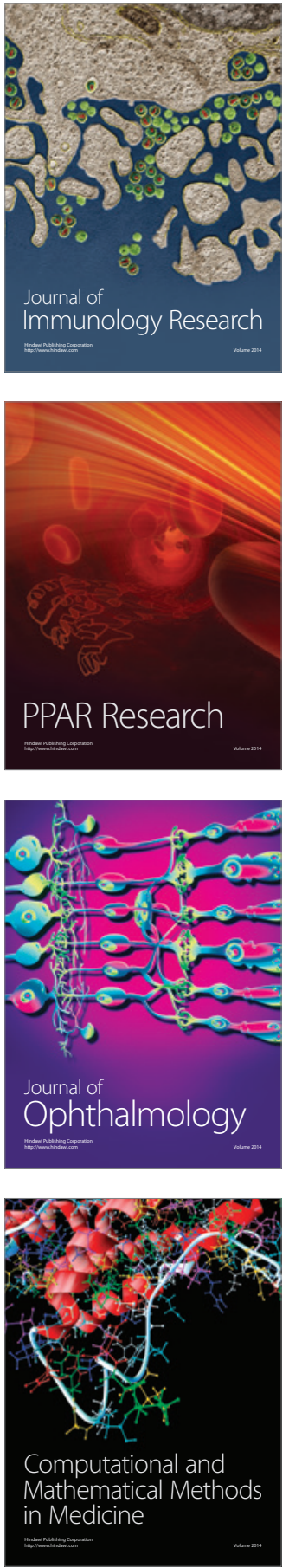

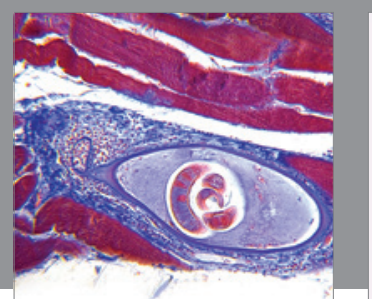

Gastroenterology Research and Practice

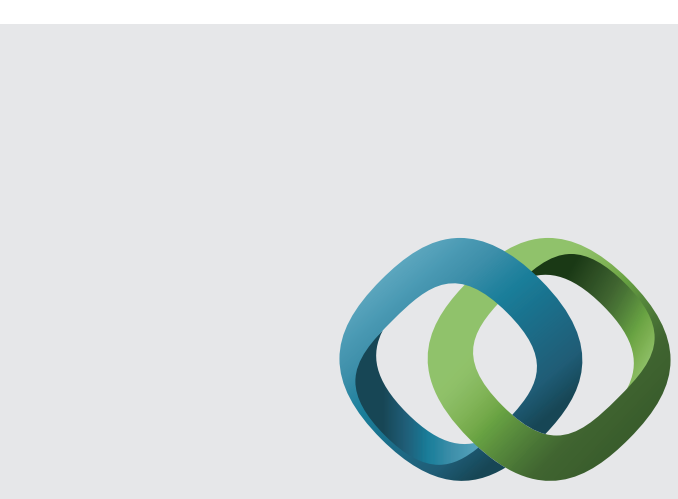

\section{Hindawi}

Submit your manuscripts at

http://www.hindawi.com
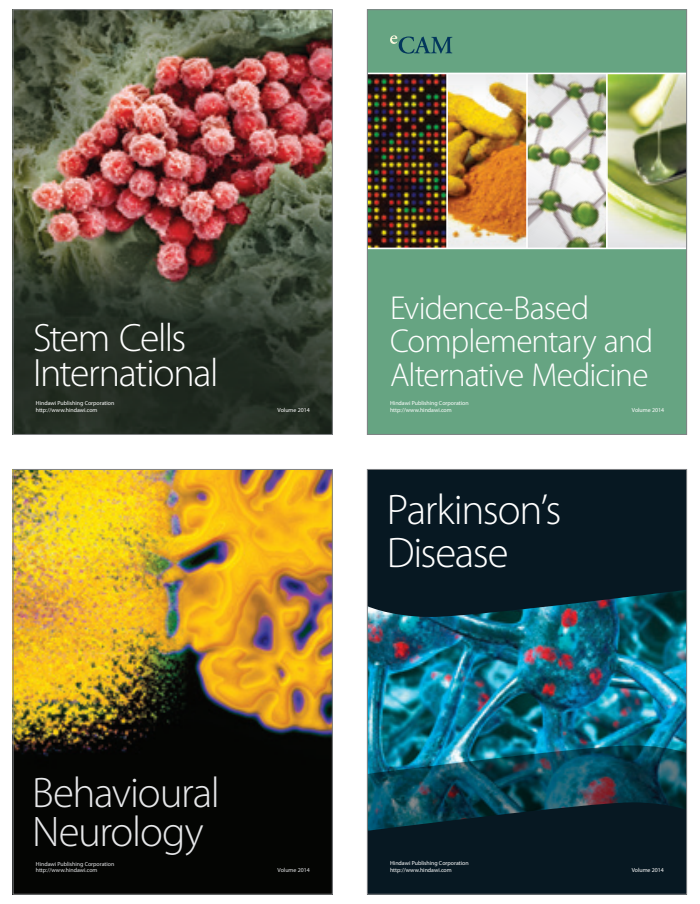
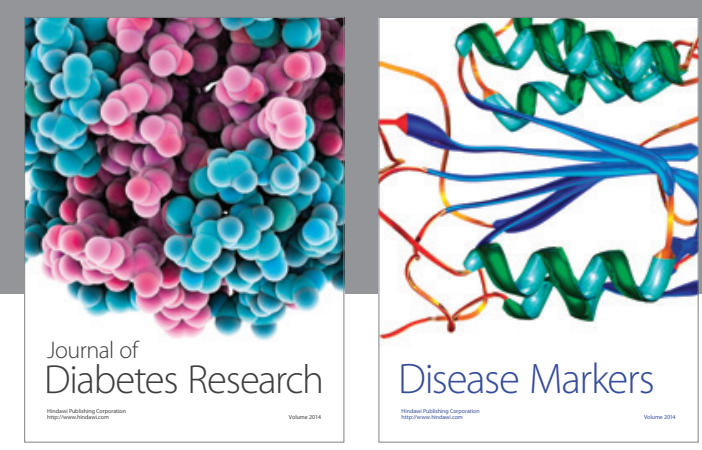

Disease Markers
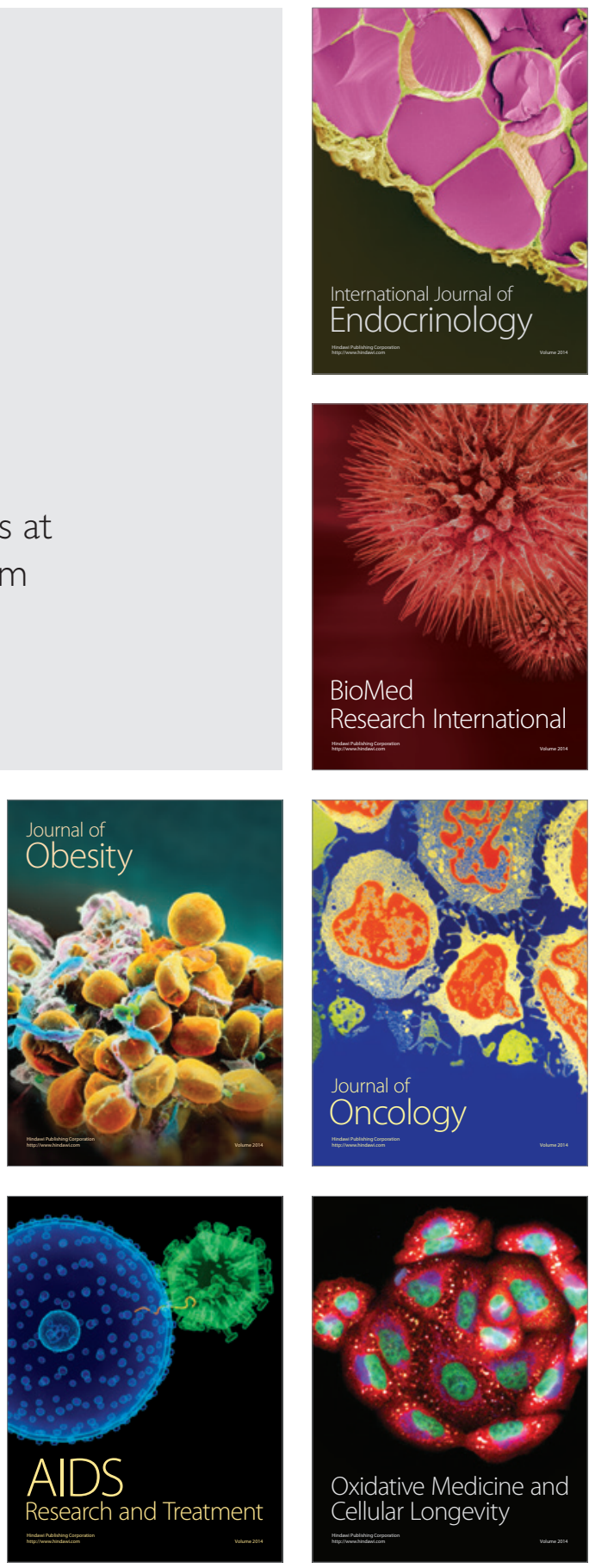